\title{
Passive Control Strategy for Multi-Tethered Tetrahedral Formation for Multipoint Scientific Measurements in LEO
}

\author{
Basel Omran $^{1}$, Dmitry Pritykin ${ }^{1}$ \\ ${ }^{1}$ Skolkovo Institute of Science and Technology \\ Moscow, Russia \\ basel.omran@skoltech.ru,d.pritykin@skoltech.ru
}

\begin{abstract}
This paper is devoted to the dynamics of a multi-tethered tetrahedral satellite formation. The proposed formation consists of a spinning tethered triangle and a free-flying spacecraft orbiting at LEO. It is shown that a proper choice of the spin rate of the tethered triangle causes it to change its orientation due to gravity-gradient torque and track the free-flying satellite that changes its periodic inertial position because of the argument of perigee drift. Furthermore, the design of J2-invariant relative orbits is utilized to minimize any undesired drift. The proposed passive control strategy results in producing high-quality tetrahedra. The proposed formation is suitable for scientific missions where three-dimensional multi-point measurements are required.
\end{abstract}

Keywords: multi-tethered tetrahedral formation, tethered triangle, formation quality, J2-invariant

\section{Introduction}

Spacecraft formations have been extensively studied throughout recent years. Multi-satellite missions provide the possibility for various applications that cannot be implemented using a single spacecraft. Several applications require spacecraft to be distributed in three-dimensional formations. One example is a mission to collect three-dimensional simultaneous multi-point measurements. The so-called curlometer technique serves as a clear explanation in which a linear estimation of Ampere's law allows deriving the strength and orientation of current density directly from the magnetic field and its gradients [1]. This technique calls for a minimum number of four spacecraft distributed in a tetrahedral formation to obtain the current density vector [1]. More importantly, each pair of the four spacecraft needs to fly equidistant from each other forming nearly regular tetrahedra along the orbit [1].

Scientific missions (e.g. Cluster II and MMS) have utilized three-dimensional formations for scientific measurements. However, strict requirements may hinder the use of formations in which a considerable amount of fuel needs to be spent for their maintenance. Analytical studies (e.g. [2]) have been conducted to construct nearly regular tetrahedral formation, and sets of initial conditions that generate optimized orbital configurations of free tetrahedral formation were found. Quality factors have been introduced that measure on the scale from 0 to 1 how close the shape of the tetrahedron is to a regular one. The best orbital configuration found in [2] for a free four-satellite formation guarantees the quality factor along the orbit to be not lower than 0.6. Our study investigates if adding tethered connections to the formation helps in maintaining the required geometry and spending less fuel.

Many researchers have investigated the dynamics and control of multi-tethered satellite formations. In particular, the study of the dynamics of out-of-plane tethered triangles. The main reason behind that is the need to obtain stable configurations suitable for Earth remote sensing missions, in which the formation plane remains oriented towards Earth. One of the major contributions was made by DeCou [3], who investigated the effect of gravity gradient on multi-body tethered formations, including the equilateral triangle. He concluded that such stable configurations could be obtained under the condition that the formation's spin rate is much higher than the orbital mean motion. Such formations can be used in deep space missions since the spin axis approximately maintains inertial orientation. Another contribution was made by Tragesser [4], who investigated the possibility to obtain a stable configuration of the near-Earth oriented spinning triangle based on the conical Linkins-Pringle equilibria with a cone angle of $46.6^{\circ}$ and a spin rate of $1.375 \mathrm{n}$ ( $\mathrm{n}$ is the mean motion). He concluded that no stable formation could be found after his proposed formation collapsed due to the slow spin rate being used. Another contribution by Pizarro and Misra [5] who investigated multi-tethered formations comprising more than three bodies. They 
found that some configurations can be stable under a high relative spin rate, at least for a short-term study. Our study utilizes the spinning tethered out-of-plane triangle for a different application, not the one being proposed for deep space missions nor Earth-oriented missions.

\section{Mission Model}

This section presents the formation setup, reference frames we use, the assumptions and equations of motion and the quality factor governing the formation design.

\section{Formation Setup}

- the formation targets a polar, near-circular orbit with an altitude of $500 \mathrm{~km}$;

- the inter-satellite distances are of the order of $500 \sim 1000 \mathrm{~m}$;

- the formation comprises four identical spacecraft forming a tetrahedron;

- three of the four spacecraft (S1, S2, S3) form a spinning tethered triangle, the fourth spacecraft (FS) is free (Fig. 1a);

- orbital configuration design is such that the formation shape is as close to a regular tetrahedron as possible.

\section{Reference Frames}

Four coordinate reference frames are used in our analysis:

- the Earth-centered inertial J2000 reference frame (N)

This frame's origin is at the Earth's center with a triad $\mathrm{N}\{\hat{X}, \hat{Y}, \hat{Z}\}$. The first axis points towards the vernal equinox, the third axis is aligned with the spin axis of Earth and the second axis completes the right-handed coordinate system (Fig. 1b).

- the orbital reference frame $(\mathrm{O})$

This frame's origin is at the center of the tethered triangle with a triad $\mathrm{O}\{\hat{r}, \hat{v}, \hat{h}\}$. The first axis is aligned with the orbital radius pointing away from Earth, the third axis is normal to the orbital plane, and the second axis completes the triad (Fig. 1c).

- the formation frame $(\mathrm{F})$.

The origin is located at the center of mass of the equilateral triangle with a triad $\mathrm{F}\{\hat{s}, \widehat{m}, \hat{n}\}$. The first axis is the spin axis of the triangle, the second axis points towards an arbitrarily chosen spacecraft ( $\mathrm{S} 1$ ), and the third axis completes the triad (Fig. 1c).

- the quasi-inertial frame (S)

This frame has a triad of $S\{\hat{x}, \hat{y}, \hat{z}\}$. Its origin coincides with the center of mass of the triangle. Initially, it is aligned with the orbital frame. The first axis is aligned with Earth's spin axis. The second axis is aligned with the $-X$-axis of the Earth-centered inertial frame, and the third axis completes the triad and aligned with the $-Y$-axis of the Earth-centered inertial frame. However, while the Earth-centered inertial frame is by definition centered at the Earth's center, this frame's center moves along the orbit (Fig. 1c).

\section{Assumptions}

Our analysis relies on the three principal assumptions, which are as follows:

- all four spacecraft are modelled as point masses;

- all tethers are massless, viscoelastic, and non-conducting; their nominal length is fixed;

- the tethers accommodate tension force when the distance between a pair of tethered spacecraft is greater than the tether's nominal length. No compressive forces are present. 

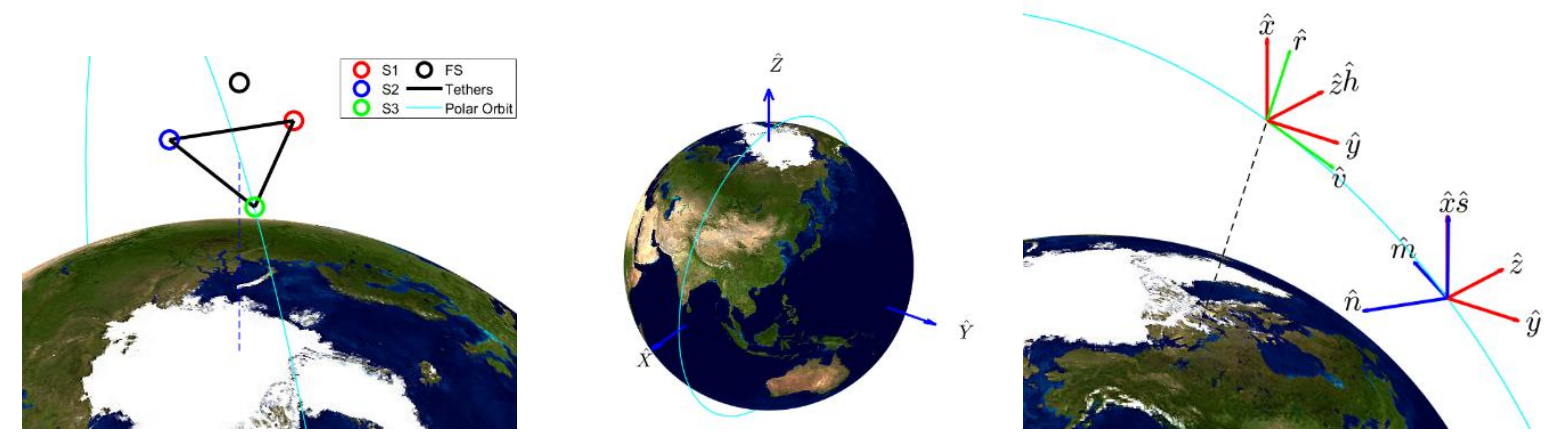

Fig. 1 a) Formation setup: a spinning tethered triangle with a free-flying spacecraft. b) the Earth-centered inertial reference frame. c) the orbital frame (green), the formation frame (blue), and the quasi-inertial frame (red).

\section{Equations of motion}

The tethered satellite's motion is described by a set of equations of motion relative to the orbital frame taking into account $\mathrm{J} 2$ perturbation and tether forces [6]. The dynamics is described for each satellite position vector $r_{j}=\left(x_{j} y_{j} z_{j}\right)^{T}$ as:

$\ddot{x}_{j}=2 \dot{y}_{j} \omega_{z}-x_{j}\left(n_{j}^{2}-\omega_{z}^{2}\right)+y_{j} \alpha_{z}-z_{j} \omega_{x} \omega_{z}-\left(\gamma_{j}-\gamma\right) s_{i} s_{\theta}-r\left(n_{j}^{2}-n^{2}\right)+\frac{1}{m}\left(T_{j x}^{a 1}+T_{j x}^{a 2}\right)$,

$\ddot{y}_{j}=-2 \dot{x}_{j} \omega_{z}+2 \dot{z}_{j} \omega_{x}-x_{j} \alpha_{z}-y_{j}\left(n_{j}^{2}-\omega_{z}^{2}-\omega_{x}^{2}\right)+z_{j} \alpha_{x}-\left(\gamma_{j}-\gamma\right) s_{i} c_{\theta}+\frac{1}{m}\left(T_{j y}^{a 1}+T_{j y}^{a 2}\right)$,

$\ddot{z}_{j}=-2 \dot{y}_{j} \omega_{x}-x_{j} \omega_{x} \omega_{z}-y_{j} \alpha_{x}-z_{j}\left(n_{j}^{2}-\omega_{x}^{2}\right)-\left(\gamma_{j}-\gamma\right) c_{i}+\frac{1}{m}\left(T_{j z}^{a 1}+T_{j z}^{a 2}\right)$,

where the subscript $j=1,2,3,4$ designates each satellite, $m$ is the identical mass of each tethered satellite, $\omega_{x}, \omega_{z}, \alpha_{x}, \alpha_{z}$ are the components of the angular velocity and the angular acceleration of the orbital frame, $s_{i}, s_{\theta}, c_{i}, c_{\theta}$ are the sine and cosine of the inclination $i$ and the true latitude $\theta$, and $\gamma, \gamma_{j}, n, n_{j}$ are auxiliary quantities. All relations for these parameters are given in Appendix A, Eqs. (A.1) - (A.11). Furthermore, all time-varying parameters in Eqs. (1) are functions of five variables $(r, v, h, i, \theta)$ that describe the motion of the orbital frame's center of mass. The five differential equations of the center of mass are given in Appendix A, Eqs. (A.12) - (A.16). Note that the last terms of Eqs. (1) are tension forces applied only to tethered satellites from their two tethered neighbours denoted by superscripts $a 1$ and $a 2$. The tension force is given by the massless visco-elastic model:

$$
T_{j}^{a \vartheta}=\left\{\left[k\left(\left|r_{j}-r_{a \vartheta}\right|-l_{0}\right)+b \frac{d}{d t}\left|r_{j}-r_{a \vartheta}\right|\right] \frac{r_{j}-r_{a \vartheta}}{\left|r_{j}-r_{a} \vartheta\right|}, \text { if }\left|r_{j}-r_{a \vartheta}\right|>l_{0} 0,\right. \text { otherwise, }
$$

where $r_{j}$ and $r_{a \vartheta}$ are the position vectors of two tethered satellites, $\vartheta=1,2$ designates each tethered neighbouring satellite, $k$ and $b$ are the spring and damping coefficients respectively, and $l_{0}$ is the nominal length of the tether.

\section{Quality factor}


It is essential to assess the quality of tetrahedra formed by spacecraft formations along their orbits. As discussed earlier, the shape of the tetrahedron directly affects the accuracy of scientific measurements. Different quality factors have been proposed [7,8] before; however, we shall employ the so-called Robert-Roux parameter [7] given by:

$$
Q_{r}=\left(\frac{9 \pi}{2 \sqrt{3}} \frac{v_{t}}{v_{s}}\right)^{\frac{1}{3}}
$$

where $v_{t}$ is the true volume of the tetrahedron and $v_{s}$ is the volume of the sphere that circumscribes the tetrahedron. This factor takes a range of values between 0 and 1 . When the distance between each spacecraft pair is equal, the quality factor is one, and the tetrahedron becomes regular. When all spacecraft belong to the same plane, the tetrahedron degenerates, and its quality factor is zero. The Robert-Roux parameter choice is based on its superiority in determining the spatial gradient of the magnetic field as discussed in detail in [7], and it is used in the Cluster Science Data System [8].

\section{Orbit Design and Initial Conditions}

The formation is designed based on orbital elements. Table 1 presents the osculating orbital elements of the triangle's center of mass and the free-flying spacecraft.

Table 1: Orbit design using orbital elements for the center of the triangle and the free-flying spacecraft.

\begin{tabular}{|c|c|c|c|c|c|c|}
\hline CoM & $a[\mathrm{~m}]$ & $e[-]$ & $i[\mathrm{deg}]$ & $\Omega[\mathrm{deg}]$ & $\omega[\mathrm{deg}]$ & $M_{0}[\mathrm{deg}]$ \\
\hline Free SC & 687813739.3 & $2.967 \cdot 10^{-5}$ & 90 & 0 & 90 & 0 \\
\hline $\begin{array}{c}\text { The rationale } \\
\text { for the } \\
\text { absolute } \\
\text { values }\end{array}$ & LEO & $\begin{array}{c}\text { Near } \\
\text { circular } \\
\text { orbit }\end{array}$ & Polar orbit & Irrelevant & $\begin{array}{c}\text { Starting at the North } \\
\text { pole }\end{array}$ \\
\hline $\begin{array}{c}\text { The rationale } \\
\text { for the } \\
\text { relative } \\
\text { values }\end{array}$ & $\begin{array}{c}\text { J2- } \\
\text { invariant }\end{array}$ & $\begin{array}{c}\text { Height of } \\
\text { the regular } \\
\text { tetrahedron }\end{array}$ & $\begin{array}{c}\text { No desired } \\
\text { out-of-plane } \\
\text { motion }\end{array}$ & $\begin{array}{c}\text { No desired } \\
\text { out-of- } \\
\text { plane } \\
\text { motion }\end{array}$ & $\begin{array}{c}\text { Opposite locations and } \\
\text { close relative orbits }\end{array}$ \\
\hline
\end{tabular}

The semimajor axis of the free-flying satellite was adjusted to obtain J2-invariant relative orbits. The sum of eccentricity is obtained by dividing the initial regular tetrahedron's height by the semimajor axis to obtain a regular tetrahedron initially.

There are 29 initial conditions needed for solving the differential equations presented in Eqs. (1) and Eqs. (A.12) (A.16). Five initial conditions are necessary for the center of the orbital frame, 12 for the four satellites' positions, and 12 for their velocities. The five initial conditions of the center of the orbital frame are obtained based on the orbital elements given in Table 1 . Note that the dynamics of $\mathbf{J} 2$ is axially symmetric, and therefore it is independent of the right ascension of ascending node, $\Omega$.

Table 2: Initial conditions of the center of mass of the triangle (orbit frame's center).

\begin{tabular}{|c|c|c|c|c|c|}
\hline Initial condition & $r[\mathrm{~m}]$ & $v[\mathrm{~m} / \mathrm{s}]$ & $h\left[\mathrm{~m}^{2} / \mathrm{s}\right]$ & $i[\mathrm{deg}]$ & $\theta[\mathrm{deg}]$ \\
\hline Value & 6877933 & $\approx 0$ & $5.236 \cdot 10^{10}$ & 90 & 90 \\
\hline
\end{tabular}


The Earth-facing equilateral triangle of a side length $l_{0}$ is centered at the orbital frame's center, with its height initially aligned with the along-track axis. The velocity of each satellite in the triangle is obtained by the cross product of the formation's angular velocity relative to the orbital frame with each position. The free-flying satellite's initial positions and velocities are obtained first by converting its orbital elements from Table 1 to the Earth-centered inertial frame and then to the orbital frame using the rotation matrix that relates both frames. The initial positions and velocities of the four satellites are given in Table 3. The design parameters used in the numerical study are presented in Table. 4.

Table 3: Initial conditions of the four satellites.

\begin{tabular}{|c|c|c|c|c|}
\hline & S1 & S2 & S3 & FS \\
\hline$r_{j}[\mathrm{~m}]$ & $(0,-144.337,250)^{T}$ & $(0,-144.337,-250)^{T}$ & $(0,288.675,0)^{T}$ & $(410.543,0,0)^{T}$ \\
\hline$v_{j}[\mathrm{~m} / \mathrm{s}]$ & $(0,-5.036,-2.907)^{T}$ & $(0,5.036,-2.907)^{T}$ & $(0,0,5.815)^{T}$ & $(0,-0.9075,0)^{T}$ \\
\hline \multicolumn{4}{|c|}{ Table 4: Design parameters of the tethered triangle. } \\
\hline$l_{0}[\mathrm{~m}]$ & $m[\mathrm{~kg}]$ & $k[\mathrm{~N} / \mathrm{m}]$ & $b[\mathrm{~kg} / \mathrm{s}]$ & $\omega_{F / O}[\mathrm{rad} / \mathrm{s}]$ \\
\hline 500 & 12 & 10 & 0 & $(0.02014,0,0)^{T}$ \\
\hline
\end{tabular}

\section{Numerical Simulation}

This section presents the simulation results of the spinning tethered triangle dynamics, the tetrahedral formation dynamics, and a passive control strategy to maintain the formation. The tetrahedral formation is simulated by numerically integrating the equations of motion (1). The initial phase of the design is conducted in the central gravity field by dropping all $\mathbf{J} 2$ terms from the nonlinear equations of motion. In the second phase of the design, the nonlinear equations of motion, including J2, are employed to simulate the tetrahedral formation's complete dynamics.

\section{Dynamics of the Out-Of-Plane Spinning Tethered Triangle}

The triangle forms the base of the tetrahedron, and therefore its dynamics needs to be investigated before proceeding to construct the tetrahedron. The tethered triangle dynamics is best described by the following three parameters: the extension of tethers, the in-plane angle $\alpha$, and the out-of-plane angle $\beta$. The angle between the x-axis of the quasi-inertial plane and the spin axis projection onto $x-y$ is denoted by $\alpha$ (Fig. 3). The angle the spin axis makes with the $x-y$ quasi-inertial frame is denoted by $\beta$ (Fig. 3).

The main factor that affects all three parameters is the spin rate of the triangle relative to the orbital frame. Let us introduce the dimensionless factor $r_{s}$ as follows:

$$
r_{s}=\frac{\omega_{F / O}}{n}
$$

where $\omega_{F / O}$ is the formation frame's angular velocity relative to the orbital frame, and $n$ is the mean motion. Note this dimensionless factor will be further referred to as spin rate.

Our numerical simulations show that the minimum spin rate needed to keep the tethers of the out-of-plane triangle taught is approximately 1.95. The out-of-plane angle is bounded with different precession amplitudes for spin rates larger than the critical value. The in-plane angle exhibits both drift and precession with varying amplitudes for different spin rates. Fig. 4 and Fig. 5 show the elongation in tethers, the out-of-plane angle, and the in-plane angle for two different spin rate values. 


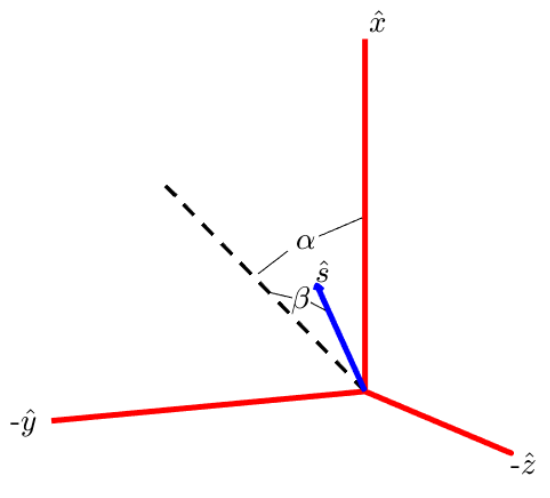

Fig. 3 Angles of spin axis: in-plane angle $\alpha$. out-of-plane angle $\beta$.

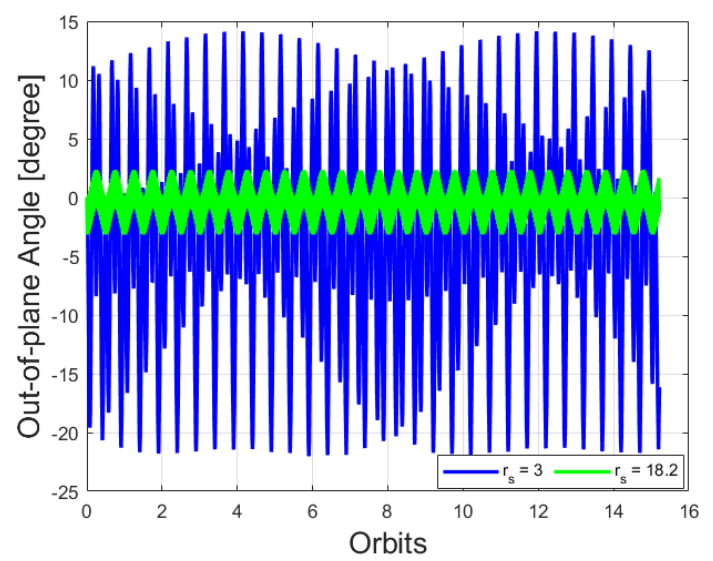

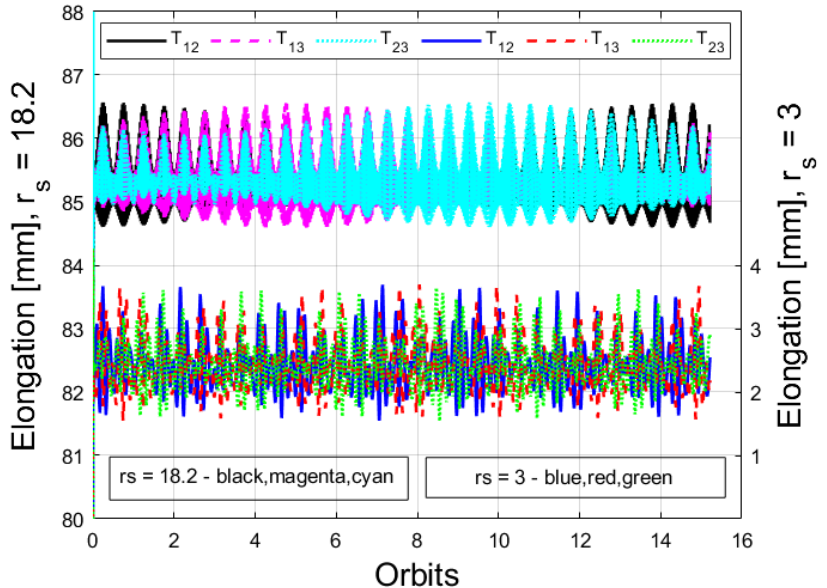

Fig. 4 Elastic elongation in the tethers for two values of spin rate: 3 and 18.2.

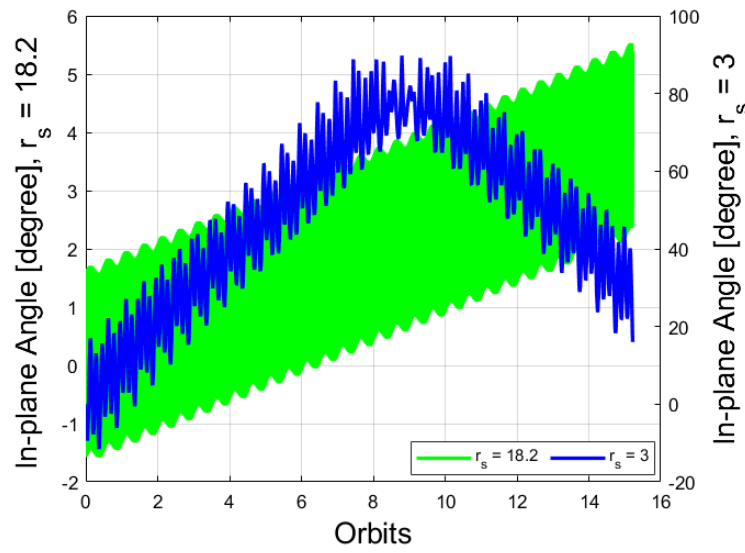

Fig. 5 Dynamics of the spin axis for two spin rate values: 3 and 18.2: a) out-of-plane angle $\beta$. b) in-plane angle $\alpha$.

Fig. 4 shows that a higher spin rate (18.2) leads to a greater extension in the tethers $(85.5 \mathrm{~mm})$. Nevertheless, a relatively low spin rate (3) is sufficient to keep the formation's shape with a low extension $(3 \mathrm{~mm})$. Both simulations use the same parameters and initial conditions except for the spin rate.

While the shape of the formation is maintained, the orientation is not; this can be deduced from Fig. 5. At a low spin rate, the out-of-plane angle (Fig. 5a) exhibits precession of higher amplitude $\left(-22^{\circ}-25^{\circ}\right)$ than in the higher spin counterpart $\left(-3^{\circ}-2.5^{\circ}\right)$. It is worth mentioning that regardless of how low spin rate is given to the formation, the out-of-plane angle's precession is bounded. In contrast, the in-plane angle dynamics exhibits both drift and precession (Fig. 5b). The precession is similar to that of the out-of-plane angle (inversely proportional to the spin rate), whereas different spin rate values result in different amounts of drift. A spin rate of 3 causes the triangle to rotate $160 \%$ day. In comparison, a high spin rate of 18.2 results in an average drift of 3.8\% day. No matter how high spin rate is given to the formation, the spin axis still exhibits drift from its inertial orientation. This result plays a significant role in the subsequent design of the tetrahedral formation. 


\section{Dynamics of the Multi-Tethered Tetrahedral Formation}

Let us now proceed to construct the tetrahedron. The relative orbits of the center of mass of the triangular formation and the free-flying spacecraft are designed based on orbital elements presented in table 1. The triangle's spin rate is purposely chosen to be 18.2 (as discussed in the following subsection)

The best way to interpret the simulation results is by analyzing the quality factor Eq. (3) shown in Fig. 6. As shown in Fig. 6a, the distance between each pair of spacecraft in the tethered triangle (black and yellow colors covered by the magenta) is maintained at a constant value $(500 \mathrm{~m}$ ). At the same time, the distance between each tethered satellite and the free-flying satellite varies along the orbit. The orbit's starting point is above the North Pole, and the initial conditions correspond to a regular tetrahedron (shown in green, Fig. 6b). As the formation orbits towards the equator, it assumes different tetrahedral shapes with quality factors ranging between 1 and 0.84 (0.88 in cyan, Fig. 6b). Note that the value of 0.84 (shown in red, Fig. 6b) corresponds to a tetrahedron of the same base and double-height. As the formation flies towards the South Pole, the same range of quality factors will be obtained in reverse (0.84 to 1$)$. This behavior in the first half of the orbit is repeated during the second half.

However, this behavior is not exactly periodic over one day, which can be explained by Fig. 5b. The drift of the triangle's spin axis due to gravity-gradient torque causes the tetrahedron to degenerate in the central gravity field. In other words, the triangular plane will not be facing the free-flying satellite with time; this is addressed in the following section by proposing a passive control strategy to keep the tetrahedral formation from degeneration.
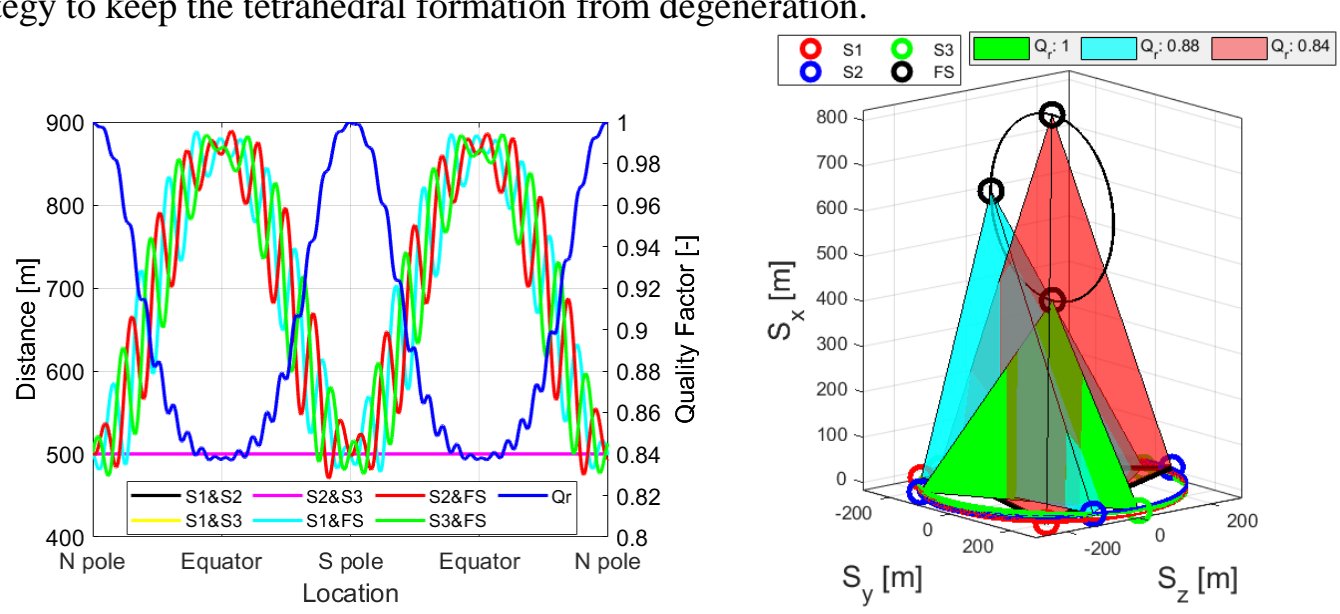

Fig. 6 The evolution of the tetrahedral formation along one orbit: a) inter-satellite distance and the quality factor. b) green: Regular tetrahedron of quality one, cyan: a tetrahedron of a quality factor of 0.88 , red: double-height tetrahedron of quality 0.84 .

\section{A Passive Control Strategy for the Multi-Tethered Tetrahedral Formation}

The passive control goal is to maintain the range of quality factors shown in Fig. 6a. The dynamics of the multi-tethered tetrahedron was until now studied in the central gravity field. In LEO, the major perturbation comes from the second degree spherical harmonic of Earth's gravity J2. It is known that J2 causes secular drift to the periapsis vector. The average rate of change of the argument of perigee is given by [9]:

$$
\dot{\omega}=\frac{3 n J_{2} R_{e}^{2}}{4 p^{2}}\left(4-5 \sin \sin i^{2}\right)
$$

where $p=a\left(1-e^{2}\right)$ is the semi-latus rectum, $n$ is the mean motion, $R_{e}$ is the Earth equatorial radius, and $i$ is the inclination. Using the values of the orbital elements given in Table 1, one obtains the drift rate according to Eq. (5) to be 
approximately $3.82 \%$ day westward. Based on this value, a spin rate of 18.2 is given to the triangle to match the drift of the argument of perigee. Fig 7 shows the design and the result of the passive controller.

Fig. 7a shows a four-day simulation of the tetrahedron in the quasi-inertial frame. In this frame, two closed orbits of the free-flying satellite form one relative orbit in the orbital frame. The first relative orbit is shown in blue, while the last relative orbit after four days is shown in green. The projection of the free-flying satellite's trajectory onto the x-y plane of the quasiinertial frame is shifted with time due to the drift of the argument of perigee of its orbit. At the same time, the projection of the trajectory of the triangle onto the $x-y$ plane represented by one of its three satellites is rotated with time due to gravitygradient torque acting on its orbit. The amount of rotation needed to keep the triangle oriented towards the free-flying satellite is approximately $3.82 \%$ day to match the average rate of change of the argument of perigee; this justifies the choice of the spin rate (18.2) as seen in Fig. 7c.

Moreover, the four-day simulation shows that the drift is periodic and linear; this can be seen in Fig. 7c, in which the amount of drift after four days is four times the daily drift $\left(15.3^{\circ}\right)$. The undesired precession of the spin axis is bounded under the effect of $\mathrm{J} 2$ with an amplitude of $3^{\circ}$ in the $\mathrm{x}-\mathrm{y}$ plane (small window in Fig. 7c), and with an amplitude of $5.5^{\circ}$ out of the plane (Fig. 7b); this ensures that the triangle is passively-spin, passively-gravity-gradient stabilized.
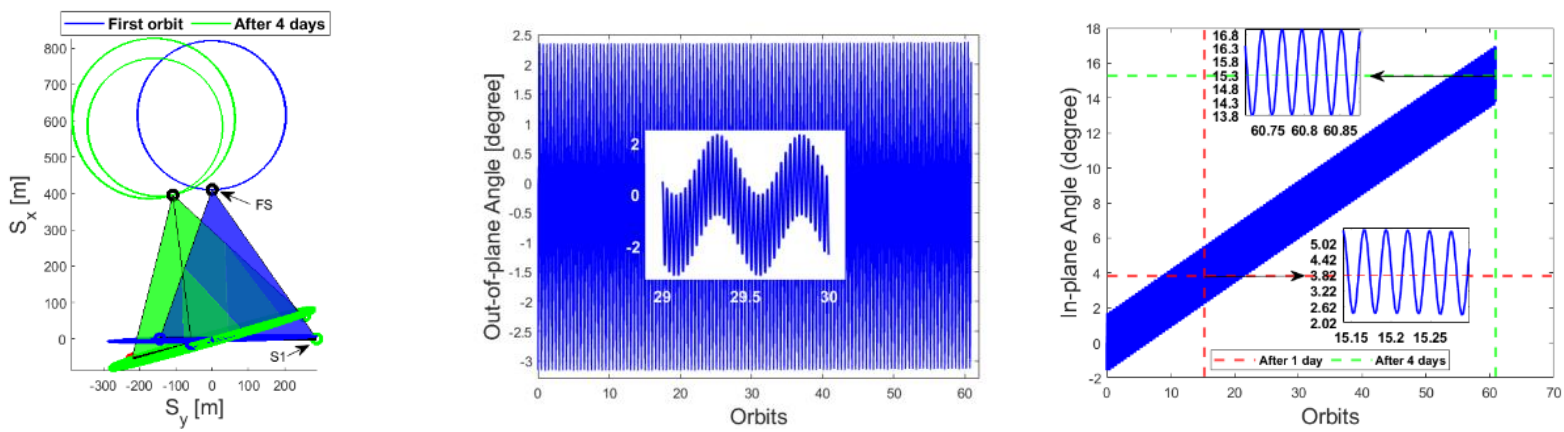

Fig. 7 A four-day simulation in the quasi-inertial frame: a) drift of the free-flying satellite and the triangle's rotation in the quasi-inertial frame. b) dynamics of the out-of-plane angle $\beta$. c) dynamics of the in-plane angle $\alpha$.

The tetrahedral formation still degenerates over time; this is due to the misalignment of the center of mass of the triangle and the free-flying satellite. In other words, the relative average drift of the angle between the position vectors of the center of mass of the triangle and the free-flying spacecraft is not zero. The design of J2-invariant relative orbits was discussed in the literature [10]. Briefly, including $\mathrm{J} 2$ in the force model makes the condition of equal mean anomaly rates and consequently equal semimajor axes no longer valid for the relative orbits to be bounded. Instead, the relative average drift of the angle between two spacecraft position vectors needs to be zero; this calls for using mean orbital elements instead of the osculating ones. Our study chose a numerical approach based on an optimizer from STK software to design such orbits; this results in a slight correction to the free-flying spacecraft orbit's semimajor axis $(a=6878.1393 \mathrm{~km})$. Fig. 8 shows the design of the J2invariant relative orbits. 

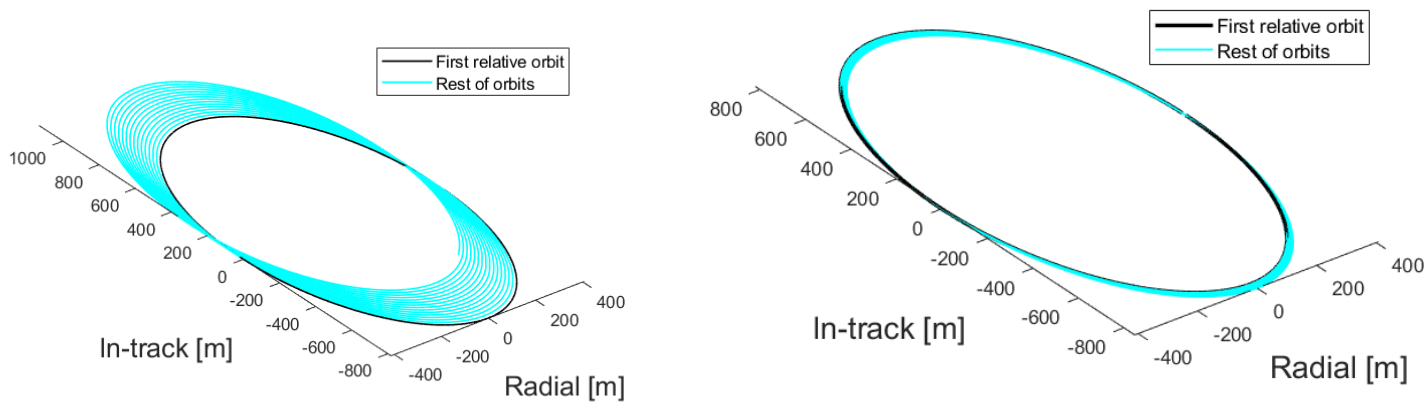

Fig. 8 Drift of the relative orbit along the track in the orbital frame. a) one-day simulation using osculating orbital elements (equal semimajor axes). b) four-day simulation using mean orbital elements (equal mean arguments of latitude).

As shown from Fig. 8a, the orbit of the free-flying satellite relative to the center of mass of the tringle drifts significantly along the track during one-day simulation when equal semimajor axes condition is imposed. In contrast, when designing the relative orbits by adjusting the semimajor axes to set the relative average drift of the angle between the center of mass and the free-flying satellite (relative argument of latitude) to zero, only a negligible drift along the track in the four-day simulation is present (Fig. 8b). Note that the use of tethers reduces the complexity of such design because only two points lying in the orbital plane (the CoM of the triangle and the free-flying satellite) need to be taken care of instead of four points in threedimensional space. Otherwise, the use of J2-invariant relative orbits would not be possible [2].

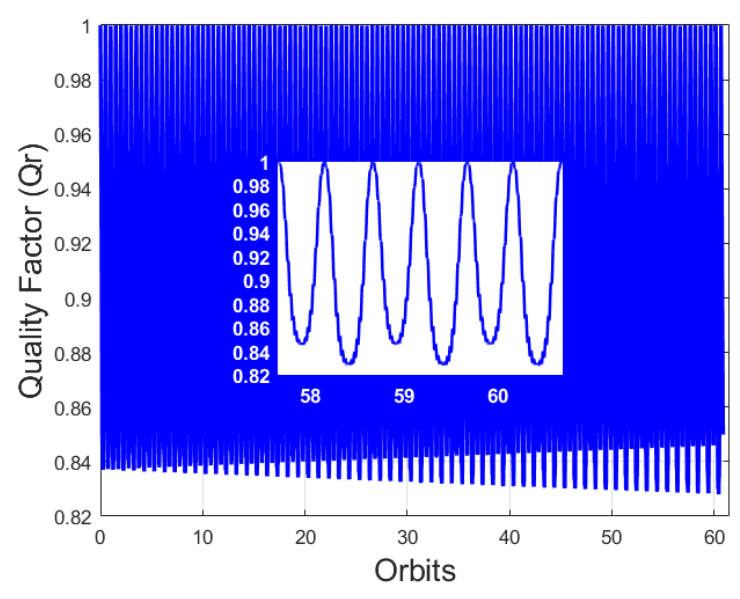

Fig. 9 The quality factor of tetrahedra formed during four days.

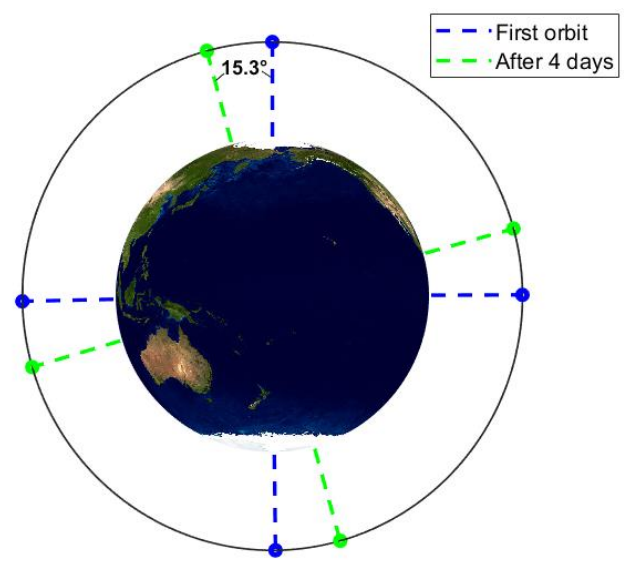

Fig. 10 Shift of tetrahedra locations.

Fig. 9 shows the quality factor for a four-day simulation. As can be seen, the tetrahedron's quality after four days has a minimum value of approximately 0.83 , a negligible drop that can be easily compensated for. From the small window in the 
figure, one can see that the local minima alternate between 0.85 and 0.83 in each orbit. This result matches the one obtained from Fig. 7a, in which the second half of the relative orbit (second quasi-inertial orbit) shrinks with time.

It is worth noting that all tetrahedra formed in the first orbit will be obtained at different locations $\left(3.82^{\circ}\right.$ westward) in each successive day. In particular, the regular tetrahedra obtained in the polar regions during the first orbit will now travel constantly to different locations of the orbit, reaching the equators after 23.5 days. Fig. 10 shows the four-day shift of the four tetrahedra formed at the poles and the equators of the first day $\left(15.3^{\circ}\right)$. To sum up, if one can imagine the orbit as a giant wall clock centered at Earth's center with its hands moving in the orbital plane, the period will be 94 days.

\section{Conclusion}

A multi-tethered tetrahedral formation consists of a spinning tethered triangle with a free-flying spacecraft is designed. The dynamics of the out-of-plane spinning tethered triangle is revised. A passive control strategy proposes a proper design choice of the spin rate of the triangle to cause it changes its attitude, due to gravity-gradient torque, at the same rate as the average rate of change of the argument of perigee, due to J2. The design of J2-invariant relative orbits is utilized to set the relative mean argument of latitude to zero and consequently minimize the drift along the track. As a result, tetrahedra of quality ranging between 1 and 0.83 are obtained in the $\mathrm{J} 2$ environment without any use of active control. The proposed formation is suitable for scientific missions where three-dimensional multi-point measurements are required.

\section{References}

[1] M. W. Dunlop, J.-Y. Yang, Y.-Y. Yang, H. Lühr, JB. Cao (2020) "Multi-spacecraft Current Estimates at Swarm," in Ionospheric Multi-Spacecraft Analysis Tools, M. W. Dunlop, H. Lühr, ISSI Scientific Report Series, vol 17. Springer, Cham. https://doi.org/10.1007/978-3-030-26732-2_5, pp. 83-90.

[2] S. Shestakov, M. Ovchinnikov, Y. Mashtakov, "Analytical Approach to Construction of Tetrahedral Satellite Formation," Journal of Guidance, Control, and Dynamics, 2019.

[3] A. B. DeCou, "Gravity Gradient Disturbances on Rotating Tethered Systems in Circular Orbit," in Proceedings of the 3rd Tethers in Space/ Toward Flight International Conference on Tethers in Space, San Francisco, CA, USA, May 1719, 1989, p. 343-351. https://doi.org/10.2514/6.1989-1593.

[4] S. Tragesser, "Formation Flying with Tethered Spacecraft," AIAA/AAS Astrodynamics Specialist Conference, Denver, CO, Aug. 2000, Paper AIAA 00-4133.

[5] Pizarro-Chong, A.K. Misra, "Dynamics of Multi-Tethered Satellite Formation," AIAA/AAS Astrodynamics Specialist Conference and Exhibit, Aug. 2000. DOI: 10.2514/6.2004-5308

[6] D. Wang, B. Wu, E.K. Poh, (2017) "Dynamic Models of Satellite Relative Motion Around an Oblate Earth," in Satellite Formation Flying, D. Wang, B. Wu, E.K. Poh, vol 87. Springer. 10.1007/978-981-10-2383-5, pp. 9-41.

[7] P. Robert And A. Roux (2000) "The Tetrahedron Quality Factors," in Analysis Methods for Multi-Spacecraft Data, Paschmann G. and Daly P. W, ISSI Scientific Report, Electronic Edition 1.1, 2000 ISSI, pp. 323-348.

[8] P. W. Daly, “The Tetrahedron Quality Factors of CSDS,” Germany, June 1994, MPAE-W-100-94-27.

[9] D. A. Vallado, Fundamentals of Astrodynamics and Applications. $4^{\text {th }}$ ed., Microcosm, inc, 2013.

[10] H. Schaub And K. T. Alfriend, "J2 Invariant Relative Orbits for Spacecraft Formations," Celestial Mechanics and Dynamical Astronomy 79, 77-95 (2001). https://doi.org/10.1023/A:1011161811472. 


\section{Appendix A}

$$
\begin{aligned}
& \omega_{x}=-\frac{k_{j 2} s_{\theta}}{h r^{3}} \\
& \omega_{z}=\frac{h}{r^{2}} \\
& r_{j}=\sqrt{\left(r+x_{j}\right)^{2}+y_{j}{ }^{2}+z_{j}^{2}} \\
& r_{j z}=\left(r+x_{j}\right) s_{\theta}+y_{j} c_{\theta}+z_{j} c_{i} \\
& -\frac{8 k_{j 2}{ }^{2} s_{i}{ }^{3} s_{\theta}{ }^{2} c_{i} c_{\theta}}{r^{6} h^{2}} \\
& \alpha_{x}=-\frac{k_{j 2} c_{\theta}}{r^{5}}+\frac{3 v k_{j 2} s_{\theta}}{r^{4} h} \\
& \alpha_{z}=-\frac{2 h v}{r^{3}}-\frac{k_{j 2} s_{i}{ }^{2} s_{2 \theta}}{r^{5}} \\
& \gamma=\frac{2 k_{j 2} s_{\theta}}{r^{4}} \\
& \gamma_{j}=\frac{2 k_{j 2} r_{j z}}{r_{j}{ }^{5}} \\
& n^{2}=\frac{\mu}{r^{3}}+\frac{k_{j 2}}{r^{5}}-\frac{5 k_{j 2} s_{i}^{2} s_{\theta^{2}}}{r^{5}} \\
& k_{j 2}=3 J_{2} \mu R_{e}{ }^{2} / 2 \\
& \frac{d r}{d t}=v \\
& \frac{d v}{d t}=-\frac{\mu}{r^{2}}+\frac{h^{2}}{r^{3}}-\frac{k_{j 2}}{r^{4}}\left(1-3 s_{i}{ }^{2} s_{\theta}{ }^{2}\right) \\
& \frac{d h}{d t}=-\frac{k_{j 2} s_{i}{ }^{2} s_{2 \theta}}{r^{3}} \\
& \frac{d i}{d t}=-\frac{k_{j 2} s_{2 i} s_{2 \theta}}{2 h r^{3}} \\
& n_{j}^{2}=\frac{\mu}{r_{j}{ }^{3}}+\frac{k_{j 2}}{r_{j}{ }^{5}}-\frac{5 k_{j 2} r_{j z}^{2}}{r_{j}{ }^{7}} \\
& \frac{d \theta}{d t}=\frac{h}{r^{2}}+\frac{2 k_{j 2} c_{i}^{2} s_{\theta}{ }^{2}}{h r^{3}}
\end{aligned}
$$

